\title{
DIFERENCIAS EN FUNCIÓN DEL SEXO EN LA PERCEPCIÓN DEL RIESGO ASOCIADO AL CONSUMO DE DROGAS EN JÓVENES
}

\author{
SEX DIFFERENCES IN THE PERCEPTION OF RISK \\ ASSOCIATED WITH DRUG USE IN YOUNG
}

\author{
CARLOS HERRUZO ${ }^{1}$, VALENTINA LUCENA ${ }^{1}$, \\ ROSARIO RUIZ-OLIVARES ${ }^{1}$, ANTONIO RAYA ${ }^{1}$ Y \\ MARÍA JOSÉ PINO ${ }^{1}$.
}

Cómo referenciar este artículo/How to reference this article:

Herruzo, C., Lucena, V., Ruiz-Olivares, R., Raya, A. y Pino, M. J. (2016). Diferencias en función del sexo en la percepción del riesgo asociado al consumo de drogas en jóvenes [Sex differences in the perception of risk associated with drug use in young]. Acción Psicológica, 13(1), 79-90. http://dx.doi.org/10.5944/ap.13.1.17422

\begin{abstract}
Resumen
El objetivo de este estudio consiste en caracterizar la percepción del riesgo asociado tanto a sustancias legales (alcohol, tabaco e inhalantes) como ilegales (cannabis, cocaína, heroína, anfetaminas, drogas de diseño, alucinógenos) entre los jóvenes de 18 a 29 años en seis dimensiones (problemas para la salud física, salud psíquica, tráfico, capacidad para realizar tareas, relaciones interpersonales y problemas legales) y, además, si existen diferencias de percepciones en
\end{abstract}

función del sexo. Con un diseño ex post facto de grupo único se han estudiado los perfiles perceptivos de 3819 jóvenes andaluces en las nueve drogas citadas. La percepción de los jóvenes muestra patrones diferentes para cada droga, destacando que existen ciertos sesgos los problemas psíquicos derivados del alcohol, que son vistos como menos peligrosos que los problemas de salud o los efectos sobre la salud del cannabis, que se ven como menos peligrosos que los del tabaco. Con respecto a la variable sexo, las mujeres perciben las drogas estudiadas como más peligrosas, salvo el caso de los inhalantes y ciertas dimensiones específicas de otras drogas, en

Agradecimientos: este trabajo ha sido posible gracias al convenio de colaboración entre la Consejería de Igualdad y Bienestar Social de la Junta de Andalucía y la Universidad de Córdoba para el estudio de las adicciones en jóvenes.

Correspondencia: Carlos Herruzo. Grupo Comportamientos de Riesgo. Facultad de Ciencias del Trabajo, Universidad de Córdoba.

Email: z42hepic@uco.es

${ }^{1}$ Universidad de Córdoba, España.

Recibido: 07 de enero de 2016.

Aceptado: 15 de febrero de 2016. 
concreto, el riesgo para la salud física en la cocaína, y los accidentes de tráfico y el riesgo para la salud física en el caso de las anfetaminas. Se sugieren enfatizar estos aspectos en las campañas informativas y preventivas.

Palabras clave: drogas legales e ilegales; jóvenes 18-29 años; percepción de riesgo; sexo.

\begin{abstract}
The aim of this study was to characterize the perception of the risk associated to both legal substances (alcohol, tobacco and inhalants) as illegal (cannabis, cocaine, heroin, amphetamines, designer drugs, hallucinogens) among youth 18 to 29 years old in six dimensions (problems for the physical health, mental health, traffic, ability to perform tasks, interpersonal relations and legal problems), and also, if weather such perceptions are different by sex. With a ex post facto single group design, were studied the perceptual profiles of 3,819 Andalusian young people about cited drugs. The perceptions of young people show different patterns for each drug, enhancing that there are certain biases about the psychic problems derivatives of alcohol, which are viewed as less dangerous than the problems of health; or the health effects of cannabis, which are seen as less dangerous than the tobacco. With regard to sex variable, women perceive drugs studied as more dangerous, except in the case of inhalants and certain specific dimensions of other drugs, in particular, the risk to the physical health in cocaine, and traffic accidents and the risk to the physical health in the case of amphetamines. Emphasizing these aspects in information campaigns and preventive measures is suggested.
\end{abstract}

Keywords: Illegal and legal drugs; youth 18-29 years old; risk perception; sex.

\section{Introducción}

El consumo de drogas entre los jóvenes sigue siendo una importante preocupación social y desde hace décadas muchos países en todo el mundo han dedicado recursos para la puesta en marcha de observatorios o agencias que realicen periódicamente estudios, además de los dedicados al tratamiento, prevención e investigación de los problemas relacionados con dicho consumo tales como problemas en la familia y en la escuela, conducción en estado de embriaguez, discapacidades, suicidio, agresiones, violencia, delitos contra el orden público, conducta sexual de alto riesgo, etc. (Alonso, Pastor, Montoro y Esteban, 2015; Jiménez-Muro, Beamonte, Marquete, Gargallo y Nerín, 2009; La Caixa, 2015; Morales-Manrique, Bueno-Cañigral, Aleixandre-Benavente y Valderrama-Zurián, 2010).

A lo largo de estas décadas se han llevado a cabo muchas investigaciones tratando de caracterizar tanto el consumo como a los consumidores, destacando los que tratan de buscar las causas de la iniciación y mantenimiento del consumo entre los adolescentes (Cava, Murgui y Musitu, 2008; Dodge et al., 2006; Hansen y O'Malley, 1996; Tirado, Aguaded y Marín, 2009).

Aunque son muchos los factores de riesgo encontrados en estas investigaciones, varias veces se ha destacado el importante papel desempeñado por las actitudes positivas que los jóvenes muestran hacia el consumo de sustancias psicoactivas: actitudes basadas en la noción de que las drogas no plantean ningún riesgo, pero que facilitan la diversión y las relaciones sociales (Gil, 2008). En este sentido, el consumo se ha vinculado con las decisiones que los jóvenes toman acerca de su consumo, no sólo en la situación concreta ante la oportunidad o estímulo para consumir, sino también con aquellas decisiones que toman sobre el consumo, es decir, que algunas personas, teniendo en cuenta los beneficios y los riesgos que implica el uso de ciertas drogas, las rechazan a pesar de las oportunidades que pueden tener para consumirlas (Calafat, Androver, Juan y Frankze, 2008; Calafat et al., 2001; Linden y Lau-Barraco, 2014; Ruiz-Olivares, Lucena, Pino y Herruzo, 2010; Trujillo, Forns y Pérez Gómez, 2007). 
Según Becoña (2007), una de las variables que influyen en estas decisiones sobre el consumo de sustancias es la percepción de riesgo, entendida como el grado en que se atribuye a un determinado tipo de conducta una amenaza especial para la salud. Es decir, la percepción del riesgo influiría en el comportamiento concreto de una persona a través de la evaluación que realiza de diferentes aspectos intrapersonales y contextuales que pueden ser favorables o desfavorables hacia la participación en la conducta en cuestión: en este caso, el consumo de drogas.

Las mayores diferencias en la percepción de los riesgos asociados con el consumo de drogas se han relacionado más con el tipo de droga que con los patrones de consumo (Fernández et al., 2003; Ruiz-Olivares, Lucena, Pino y Herruzco, 2010; Ruiz-Olivares, Lucena, Pino, Raya y Herruzo, 2010; Scott-Parker, Watson, King y Hyde, 2013). En este sentido, las ideas preconcebidas acerca de las diferentes sustancias que, a la vez, se apoyan en la propia experiencia de consumo, en las creencias y en la construcción social de las diferentes sustancias, influirán decisivamente en su consumo (Becoña, 2000, 2007). El riesgo que la población percibe ante el uso de distintas drogas podría interpretarse como un factor de protección frente al consumo de las mismas y, de manera especial, en su inicio (EDADES, Ministerio de Sanidad, Servicios Sociales e Igualdad, 2015; OEDT, 2010). Según las encuestas nacionales ESTUDES (Encuesta Estatal de Uso de Drogas en Educación Secundaria, Ministerio de Sanidad, Servicios Sociales e Igualdad, 2013) y EDADES (Ministerio de Sanidad, Servicios Sociales e Igualdad, 2015), en España, la percepción del riesgo de consumo de sustancias legales -alcohol, tabaco y hipnosedantes- es menor que el asociado a las drogas ilegales -cannabis, cocaína y alucinógenos- (Donate et al., 2008; Ruiz-Olivares, Lucena, Pino y Herruzo, 2010; Ruiz-Olivares, Lucena, Pino, Raya y Herruzo, 2010). Así, mientras el $94 \%$ de la población de 15 a 64 años considera que los consumos de heroína, cocaína y éxtasis pueden producir muchos o bastantes problemas, con independencia de la frecuencia con que se consuman, el riesgo asociado a probar éxtasis o cocaína una o dos veces en la vida, aumenta la tolerancia y la percepción del riesgo se reduce al $78.2 \%$ de la población en el caso del éxtasis y al $76.5 \%$ en el de la cocaína. Con respecto al consumo de cinco o seis cañas o copas en fin de semana, aproximadamente la mitad de la población no considera que éste pueda acarrear muchos o bastantes problemas.

Los sucesivos informes EDADES también han destacado que, con carácter general, la percepción de riesgo es mayor entre las mujeres que entre los hombres para todas las sustancias psicoactivas y para todas las frecuencias de consumo (Armstrong, Watling, Watson y Davey, 2014). La excepción aparece con los tranquilizantes/somníferos, sustancias de consumo más prevalente entre las mujeres. En este caso, la proporción entre sexos es similar a la hora de asociar determinado riesgo al consumo esporádico de dichas sustancias.

Con respecto a la edad, aunque hay una valoración similar del riesgo del consumo habitual de heroína, cocaína, éxtasis y un paquete diario de tabaco, no obstante, el consumo de cánnabis y el de cinc o seis copas en fin de semana, es percibido con mayor tolerancia por el segmento de entre 15 y 34 años frente al más mayor ( 35 a 64 años). Además, parece que la percepción del riesgo asociado al consumo habitual de tranquilizantes va descendiendo conforme se incrementa la edad.

La importancia del estudio de la percepción del riesgo asociado al consumo de sustancias se justifica en su papel de factor de protección frente al inicio y mantenimiento del consumo, como se ha afirmado anteriormente. Pero, además como se trata de un fenómeno dinámico, que cambia a lo largo del tiempo, es necesario actualizarlo y caracterizarlo en orden a acompasar las acciones preventivas e interventivas a tales creencias o constructos sociales. Algunos autores insisten en que la investigación sobre el consumo de drogas entre los jóvenes debe centrarse en grupos específicos de la población con el fin de examinar los hábitos de consumo, las percepciones y actitudes de los grupos específicos de los jóvenes y la dinámica del consumo de drogas en esos grupos particulares, y como medio de la promoción de su conocimiento del problema y ser capaz de poner en práctica la acción y de prevención de estrategias más eficaces (Martins, Carlson, Alexandre y Falck, 2011; Uribe, Verdugo y Zacarías, 2011). 
Además, en los distintos estudios se tiende a evaluar la percepción de las consecuencias del riesgo de forma global, siendo interesante la caracterización de las consecuencias, precisamente en aras de establecer acciones preventivas específicas que aumenten la sensibilidad de la población diana a la que se dirijan.

Por tanto, el objetivo de este estudio consiste en caracterizar la percepción del riesgo asociado tanto a sustancias legales como ilegales entre los jóvenes de 18 a 29 años y, además, si existen diferencias entre tales percepciones en función del sexo.

\section{Método}

\section{Participantes}

En el estudio han participado 3819 jóvenes andaluces, con una edad comprendida entre los 18 y 29 años (media $=21.6, D T=3.63)$ de los que un $46.7 \%$ eran hombres y un $53.3 \%$ eran mujeres. Las características generales de la muestra eran: jóvenes entre 18 y 29 donde el $38.1 \%$ tenían entre $18-20$ años; el $26.0 \%$ tenían entre 21-23 años; el $17.9 \%$ tenían entre $24-26$ años; el $13.5 \%$ tenían entre $27-29$ años y el $4.6 \%$ eran mayores de 29 años. El $40.5 \%$ estudian, el $32.1 \%$ trabajan, un $13.4 \%$ estudian y trabajan los fines de semana y un $12.9 \%$ se encuentran desempleados.

\section{Instrumentos}

El cuestionario utilizado fue una adaptación del aplicado en otros trabajos de investigación con población universitaria (Lucena et al., 2013; Ruiz-Olivares, Lucena, Pino y Herruzo, 2010; Ruiz-Olivares, Lucena, Pino, Raya y Herruzo, 2010) cuyo objetivo principal era describir los patrones de consumo de sustancias ilegales (cannabis, cocaína y drogas de diseño) en función de variables como la edad, el sexo, los estudios universitarios, características familiares, etc. El cuestionario contiene varios bloques de preguntas: datos sociodemográficos, patrones de consumo y percepción de riesgo. Las preguntas referidas a los patrones de consumo se dividían por drogas (alcohol, tabaco, cannabis, cocaína, alucinógenos, heroína, anfetaminas, drogas de diseño e inhalantes), dando cuatro opciones de respuesta: nunca he consumido, alguna vez en la vida, en los 12 últimos meses y en los 30 últimos días. $\mathrm{Y}$ en cuanto a la percepción de riesgo se preguntaba por el riesgo específico de cada sustancia en seis dimensiones con una escala tipo Likert del 1 al 5, siendo 1 una percepción de bajo riesgo ("nunca causa problemas") y 5 una máxima percepción de riesgo ("siempre causa problemas"). Las seis dimensiones consultadas fueron: (a) accidentes de tráfico, (b) problemas psíquicos (ansiedad, pérdida de memoria, daños cerebrales, etc.), (c) problemas en casa o de relación con los amigos, (d) problemas legales, e) capacidad de hacer tareas y (f) provocar enfermedades crónicas o problemas de salud física.

\section{Diseño}

El estudio se ha realizado mediante un diseño ex postfacto prospectivo de grupo único. Para la predeterminación del tamaño de la muestra, se partió de la siguiente simulación realizada con el paquete estadístico EpiInfo 2000, que para un $1 \%$ de frecuencia esperada y un margen de error del 0.01 , se obtuvo que como mínimo se debía recoger la información de 1900 sujetos. Se realizó un muestreo proporcional por conglomerados con una distribución por mancomunidades.

\section{Procedimiento}

Para la recogida de datos colaboraron profesores de la Universidad de Córdoba y los coordinadores del Programa de Ciudades ante las drogas de diversas localidades de la provincia de Córdoba, que pasaron las encuestas a la población joven de un pueblo o mancomunidad en clases de ciclos formativos, lugares de trabajo, gimnasios, centros sociales o al azar. En el apartado de instrucciones del mismo se especificaba que era una encuesta sobre el consumo de diversas sustancias y algunos com- 
portamientos en población joven, cuyo objetivo era la realización de una serie de actuaciones de prevención y tratamiento ante los problemas que pueden derivarse de estas conductas. Por último, se resaltaba la importancia de su participación, informando a su vez que el tratamiento de los datos sería estadístico de forma completamente anónima.

\section{Análisis de datos}

Con la información proporcionada por los cuestionarios se elaboró una base de datos en el programa estadístico SPSS 17. 0. Se realizó un ANOVA (modelo lineal general) para observar diferencias entre los participantes a la hora de valorar la percepción de riesgo ante el consumo de cada una de las sustancias (alcohol, tabaco, cannabis, cocaína, alucinógenos, heroína, anfetaminas, drogas de diseño e inhalantes) así como para determinar si había diferencias en cuanto a la percepción de riesgo en función del sexo.

\section{Resultados}

Como el objetivo de este estudio era caracterizar la percepción del riesgo ante nueve sustancias entre jóvenes de 18 a 29 años y estudiar si existen diferencias en función del sexo, se han representado los resultados obteni-

ALCOHOL: PERCEPCIÓN DEL RIESGO DE CAUSAR PROBLEMAS

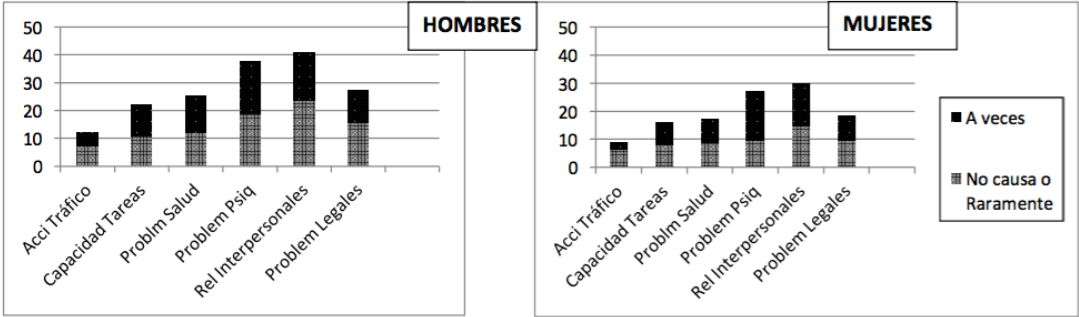

TABACO: PERCEPCIÓN DEL RIESGO DE CAUSAR PROBLEMAS

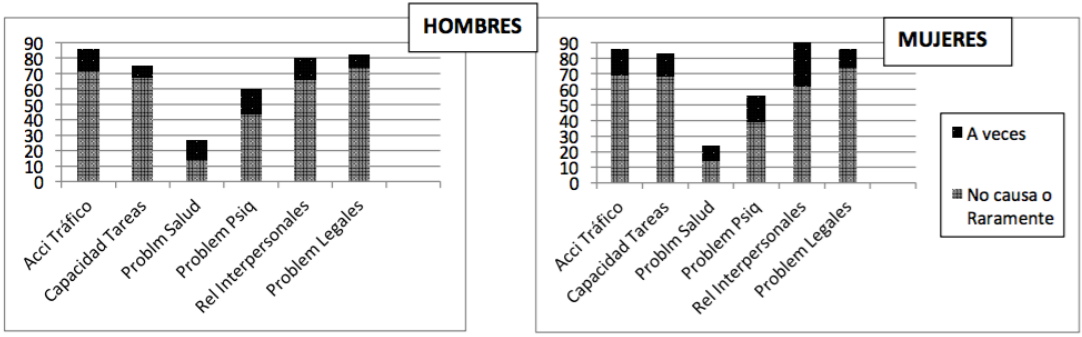

INHALANTES: PERCEPCIÓN DEL RIESGO DE CAUSAR PROBLEMAS

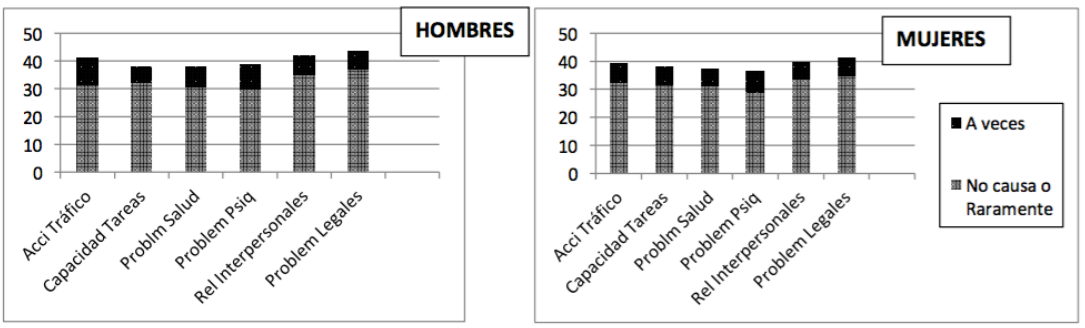

Figura 1. Percepción del riesgo de causar problemas ante el alcohol, el tabaco y los inhalantes en hombres y mujeres. 
dos en las figuras 1,2 y 3 . Como el riesgo de cada sustancia y dimensión se valoraba en una escala tipo Likert de 5 puntos, para facilitar la comprensión los datos, las respuestas 1 (No causa problemas) y 2 (Raramente causa problemas) se han agrupado, así como las de 4 (bastantes problemas) y 5 (siempre causa problemas). En la figura aparecen representados los porcentajes dentro de una misma barra, y dado que la suma siempre es $100 \%$, para enfatizar las diferencias se ha omitido la parte correspondiente a la alta percepción de riesgo (respuestas 4 y 5), de forma que a dicha opción corresponde la altura que queda hasta completar el $100 \%$.
En la Figura 1 se ha representado el perfil de riesgos para hombres (izquierda) y mujeres (derecha) en las tres drogas legales estudiadas: alcohol, tabaco y los inhalantes. En ellas se puede observar cómo el alcohol es percibido como más peligroso para la conducción $(87.5 \%$ consideran que siempre o casi siempre causa problemas) que para el resto de las dimensiones medidas como la capacidad para hacer tareas, problemas psíquicos, etc. (el ANOVA arrojó diferencias significativas en todos los casos confirmando que la percepción de cada dimensión era significativamente diferente de las demás $[p=.000])$. Las mujeres perciben un riesgo mayor que los hombres ( $p=0.000$ en todos los casos).

COCAÍNA: PERCEPCIÓN DEL RIESGO DE CAUSAR PROBLEMAS
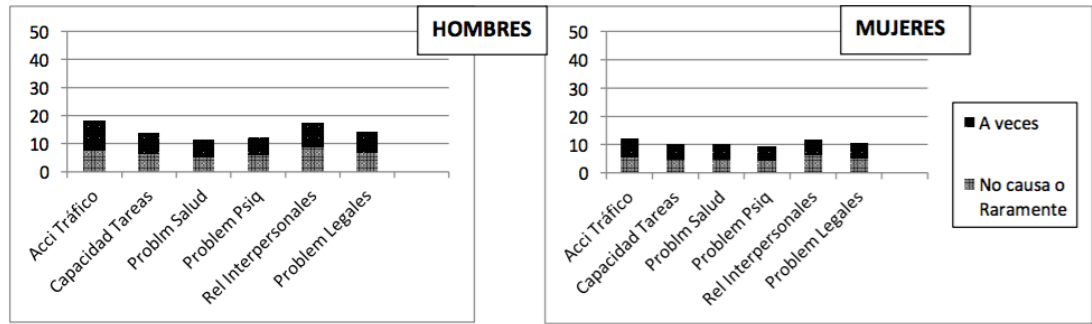

ANFETAMINAS: PERCEPCIÓN DEL RIESGO DE CAUSAR PROBLEMAS

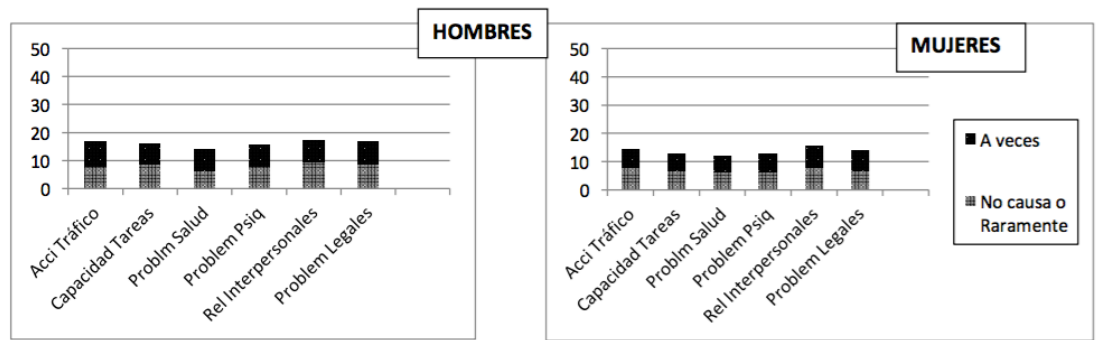

DROGAS DE DISEÑO: PERCEPCIÓN DEL RIESGO DE CAUSAR PROBLEMAS
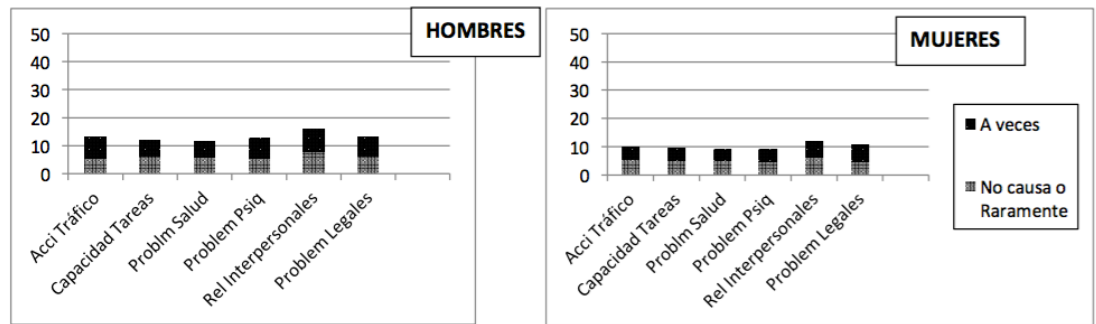

Figura 2. Percepción del riesgo de causar problemas ante la cocaína, las anfetaminas y las drogas de diseños en hombres y mujeres. 
En el caso del tabaco, la percepción de riesgo mayor corresponde a la dimensión de la salud física, y en parte a la de problemas psíquicos, siendo los demás percibidos como poco relevantes. Sólo aparecen diferencias significativas entre mujeres y hombres en problemas de salud $(p=.023)$ y en problemas psíquicos $(p=.013)$, siendo las mujeres, ligeramente superior.

En la parte inferior de la gráfica aparecen los datos correspondientes a los inhalantes. Se trata de una droga poco conocida y no aparecen diferencias significativas entre sexos estando en torno al $60 \%$ los que la consideran peligrosa.

En la Figura 2 se han representado el cannabis, los alucinógenos y la heroína. Con respecto al cannabis, el porcentaje de percepciones que no consideran peligrosas su consumo oscila entre el 25 y el $40 \%$ para los hombres y el 18 y $28 \%$ para las mujeres, siendo por tanto el riesgo percibido de las mujeres superior al de los hombres en todas las dimensiones ( $p=.000$ en todos los casos). El mayor riesgo percibido es para la salud y el menor para las relaciones interpersonales y los accidentes de tráfico (hay diferencias entre todas las dimensiones $p=.000$ ).

En el caso de los alucinógenos y de la heroína el porcentaje de personas que las perciben como peligrosas oscila en torno al $90 \%$ en todas las dimensiones, siendo superior en el caso de las mujeres frente a los hombres (los valores de p oscilan entre .002 y .01 en el caso de los alucinógenos y entre .002 y .009 en el caso de la heroína).

Por último, en la Figura 3 se han representado los resultados de la cocaína, anfetaminas y las drogas de diseño. En la parte de arriba se representan los resultados relativos a la percepción del riesgo de la cocaína. El peli-

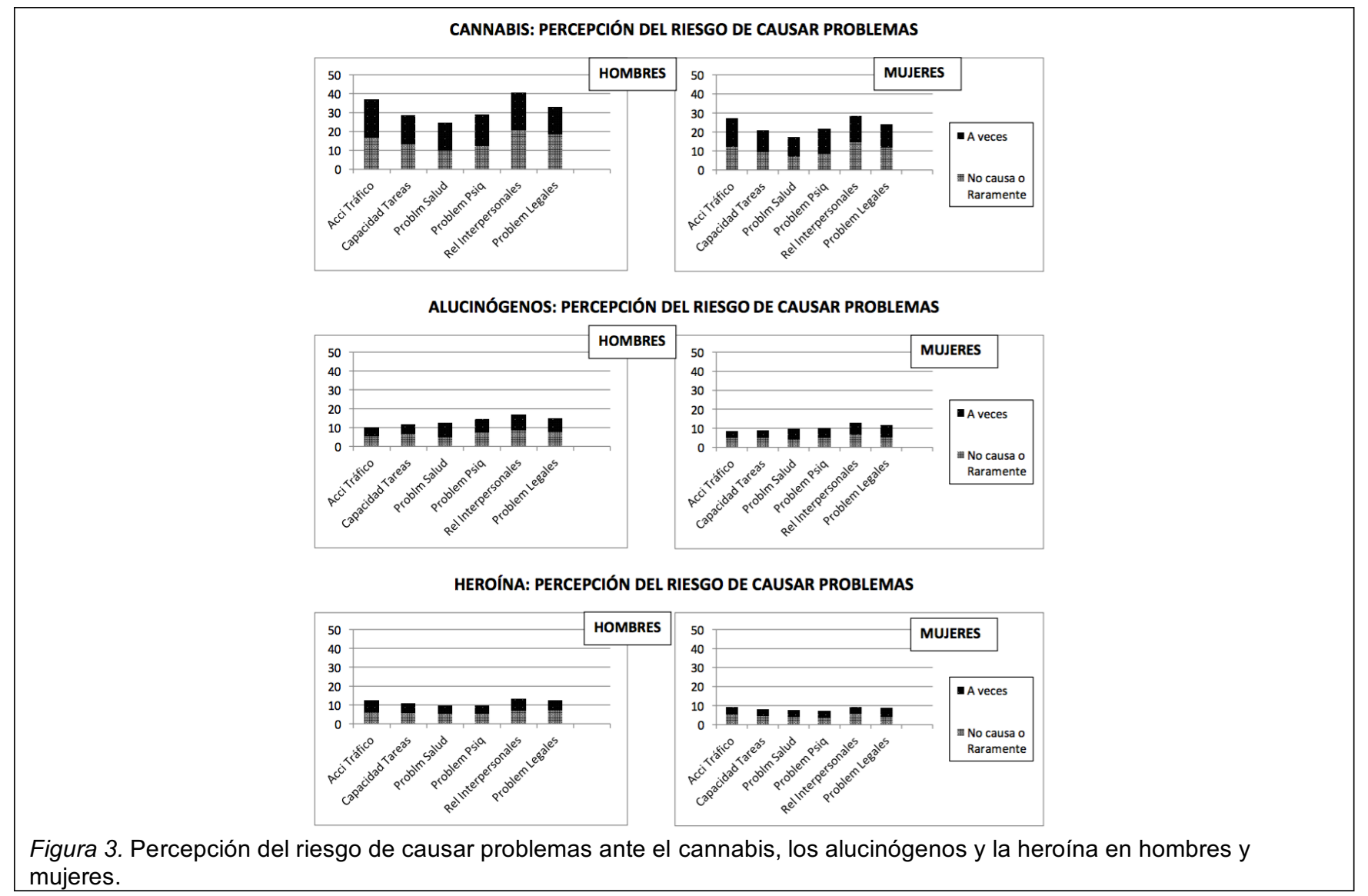


gro que se percibe mayor es para la salud física, donde no existen diferencias entre ambos sexos $(p=.065)$, aunque sí en las restantes dimensiones ( $p$ entre .000 y .005). En torno al $90.0 \%$ de las mujeres perciben que a menudo o siempre causa problemas en las 6 dimensiones, mientras que en el caso de los hombres ese porcentaje oscila entre $81.8 \%$ para los accidentes de tráfico y el $88.6 \%$ de los problemas de salud.

Las anfetaminas reciben una calificación parecida por parte de los participantes en el estudio. El mayor peligro percibido es para la salud $(85.5 \%$ de los hombres y el $87.9 \%$ de las mujeres) donde no hay diferencias significativas entre ambos sexos $(p=.105)$ ni para los accidentes de tráfico $(p=.061)$ y sí para las restantes dimensiones ( $p$ entre .001 y .039). El mayor riesgo se percibe en los problemas legales y accidentes de tráfico para los hombres $(82.9 \%)$ y las relaciones interpersonales para las mujeres $(84.4 \%)$.

Por último, las drogas de diseño presentan un perfil parecido, pero es ligeramente más alto el porcentaje de jóvenes que las perciben como peligrosas, aunque también vuelve a ser mayor la puntuación otorgada por las mujeres y el porcentaje que la califica como peligrosa (valores de $p$ que oscilan entre .000 y .009).

\section{Discusión}

Teniendo en cuenta que este estudio se proponía caracterizar la percepción del riesgo ante nueve sustancias entre jóvenes de 18 a 29 años y estudiar si existen diferencias en función del sexo, podemos decir que hemos avanzado en el conocimiento que tenemos sobre cómo perciben los jóvenes con respecto a lo que conocíamos por las encuestas nacionales, en las que el riesgo de cada sustancia se valoró de manera global. En nuestro estudio hemos medido seis dimensiones de cada sustancia, poniéndose de manifiesto que existe una clara discriminación en la percepción de los efectos en función del tipo de sustancia y el tipo de peligro. Así, en el caso del alcohol el mayor riesgo percibido es sobre los accidentes de tráfico, seguido por la capacidad para realizar tareas y los problemas de salud y legales. Sin embargo, para las relaciones interpersonales y problemas psíquicos, en el caso de los hombres, el porcentaje de los que perciben poco o ningún peligro supera el $33.0 \%$. Esto refleja un patrón que en cierta medida coincide con el énfasis que la sociedad ha puesto en destacar sus consecuencias en los accidentes de tráfico y probablemente la propia experiencia de disminuir la capacidad de realizar tareas cuando se ingiere alcohol. Sin embargo, resulta preocupante que un $10 \%$ de las mujeres y casi un $20.0 \%$ de los hombres, piensen que el alcohol no causa o pocas veces causa problemas psíquicos. Esto resulta especialmente relevante cuando los estudios ponen de manifiesto un aumento del consumo masivo (binge drinking) entre los jóvenes, cuyas principales consecuencias a largo plazo pueden manifestarse en esta dimensión. Por tanto, resultaría aconsejable aumentar el énfasis de las campañas publicitarias en estos aspectos.

En contraste, el tabaco presenta un patrón caracterizado por la percepción del peligro que implica para la salud (el $75.0 \%$ lo perciben como peligroso) seguido por lo problemas psíquicos (menos del $50.0 \%$ ), probablemente reflejando en una parte de la juventud la conciencia acerca de los problemas que genera la adicción al tabaco y los síntomas relacionados con su abstinencia como la ansiedad.

Llama la atención también el patrón perceptivo de la tercera sustancia legal estudiada, con sólo aproximadamente un $60 \%$ de los jóvenes que la consideran causante de problemas, lo que, probablemente, refleja el desconocimiento que hay entre la juventud sobre ella, dado el escaso uso de la misma en nuestro país.

Con respecto a la droga ilegal más consumida, el cannabis, el patrón perceptivo indica una tolerancia social hacia esta droga, porque el porcentaje de asignaciones como que no causa o raras veces causa problemas dobla y triplica los porcentajes de las restantes sustancias ilegales, que se hace especialmente evidente si se suman las respuestas neutras (a veces causan problemas). En este sentido, llama la atención 
algo que confirma algo destacado por EDADES, en el sentido de ser considerado menos peligroso para la salud el cannabis que el tabaco. Y también debe ser destacado el contraste de la percepción del cannabis con los alucinógenos, puesto que siendo la primera una sustancia alucinógena, el número de personas que la consideran poco o nada peligrosa o neutra es del orden de tres veces superior a la de los alucinógenos para cada una de las dimensiones estudiadas.

En el caso de la cocaína y de las anfetaminas, cuyo patrón es bastante parecido, parecen percibirse como mayores los riesgos para la salud física y psíquica. Y, finalmente, las drogas de diseño parecen mostrar un patrón parecido al de la heroína.

Con respecto a las diferencias en función del sexo, podemos afirmar que las mujeres jóvenes de 18 a 29 años, perciben un riesgo mayor en prácticamente todas las dimensiones evaluadas de siete de las nueve sustancias estudiadas. La excepción la constituye, por un lado, el tabaco en el que sólo hubo diferencias significativas entre mujeres y hombres en problemas de salud $(p=.023)$ y en problemas psíquicos $(p=.013)$, siendo la percepción de las mujeres ligeramente superior a la de los hombres. En este sentido, los resultados no coinciden con lo expresado en la encuesta EDADES, en la que la percepción de riesgo es menor en el caso del tabaco para las mujeres que para los hombres. No obstante, en dicha encuesta el riesgo se valoró con una sola pregunta sobre el riesgo general, y en la mayoría de las dimensiones medidas. Por otro lado, en los inhalantes no se encontraron diferencias significativas en función del sexo, así como en algunas de las dimensiones específicas de otras drogas, en concreto, el riesgo para la salud física en la cocaína, y los accidentes de tráfico y el riesgo para la salud física en el caso de las anfetaminas.

En definitiva, de este estudio se pueden concluir la necesidad de seguir actualizando el patrón de creencias o percepciones que los jóvenes tienen de las drogas con el objeto de enfatizar aspectos que en su visión parecen estar sesgados, como ha puesto de manifiesto este estudio, como es el caso de los problemas psíquicos derivados del alcohol o los del cannabis ya señalados.

Por último, cabe destacar de las limitaciones de este estudio. Algunas de estas limitaciones eran inherentes al diseño metodológico. Por ejemplo, la recogida de datos en el aula involucra inevitablemente un cierto grado de sesgo de selección y los resultados puede aparecer subestimados, ya que los estudiantes que asisten regularmente a clase son los que tienen los hábitos de vida más saludables (Mas et al., 2004; Ruiz-Olivares, Lucena, Pino y Herruzo, 2010; Ruiz-Olivares, Lucena, Pino, Raya y Herruzo, 2010) aunque se intentó reducir este sesgo tomando los datos en las dos primeras semanas de clase, en los que la asistencia es mayoritaria.

En futuras investigaciones, sería importante analizar las posibles diferencias en el consumo entre las poblaciones rurales no universitarias y los jóvenes universitarios con el fin de descubrir posibles sesgos perceptivos diferentes en ambos tipos de poblaciones. Por otro lado, resultaría interesante estudiar de manera específica el papel modulador del consumo en la percepción, en la línea estudiada por Pino et al. (2015) respecto a los accidentes de tráfico.

\section{Referencias}

Alonso, F., Pastor, J. C., Montoro, L. y Esteban, C. (2015). Driving under the influence of alcohol: Frequency reasons, perceived risk and punishment. Substance Abuse Treatment, Prevention, and Policy, 10(11), 1-9. http://dx.doi.org/10.1186/s13011-015$0007-4$.

Armstrong, K. A., Watling, H., Watson, A. y Davey, J. (2014). Profile of women detected drink driving via Roadside Breath Testing (RBT) in Queensland, Australia, between 2000 and 2011. Accident Analysis and Prevention, 67, 67-74. http://dx.doi.org/10.1016/j.aap.2014.02.006 
Becoña, E. (2000). Los adolescentes y el consumo de drogas. Papeles del Psicólogo, 77, 25-32.

Becoña, E. (2007). Resiliencia y consumo de drogas: una revisión. Adicciones, 19, 89- 101. Recuperado de http://search.proquest.com/docview/621792004?acc ountid $=14520$

Calafat, A., Fernández, C., Juan, M., Bellis, M. A., Bohrn, K., Hakkarainern, P., ... y Zavatti, P. (2001). Risk and control in the recreational drug culture. SONAR Project. Palma de Mallorca, España: IREFREA.

Calafat, A., Androver, D., Juan, M. y Frankze, N. (2008). Relación del consumo de alcohol y drogas de los jóvenes españoles con la siniestralidad vial durante la vida recreativa nocturna en tres comunidades autónomas en 2007. Revista Española de Salud Pública, 82(3), 323-331. Recuperado de http://scielo.isciii.es/scielo.php?pid=s1 1355727200 $8000300008 \&$ script $=$ sci_arttext

Cava, M. J., Murgui, S. y Musitu, G. (2008). Diferencias en factores de protección del consumo de sustancias en la adolescencia temprana y media. Psicothema, 20(3), 389-395. Recuperado de http://www.psicothema.com/pdf/3497.pdf

Dodge, K. A., Malone, P. S., Lansford, J. E., MillerJohnson, S., Pettit, G. S. y Bates, J. E. (2006). Toward a dynamic developmental model of the role of parents and peers in early onset substance use. En A. ClarkeStewart y J. Dunn (Eds.), Families count: Effects on child and adolescent development (pp. 104-131). New York: Cambridge University Press

Donate, I., Hevia, J. R., Gutiérrez, E., Roces, A., Redondo, N. y Álvarez, F. (2008). Informe Estudes08. Consumo de drogas en estudiantes de enseñanzas secundarias de Asturias 2008. Principado de Asturias, España: Consejería de Salud y Servicios Sanitarios. Recuperado de http://www.asturias.es/Astursalud/Ficheros/AS_Sal
ud\%20Publica/AS_Drogas/Observatorio/EstudesAst urias08_informe.pdf

Fernández, J., Álvarez, E., Secades, R., Jiménez, J. M., Cañada, A., Donate, I., ... y Vallejo, G. (2003). Consumo de drogas de síntesis en estudiantes de Secundaria del Principado de Asturias (España). Adicciones, 15, 31-37. Recuperado de http://www.adicciones.es/index.php/adicciones/articl e/view/445

Gil, J. (2008). Consumo de alcohol entre estudiantes de enseñanzas secundarias. Factores de riesgo y factores de protección. Revista de Educación, 346, 291-313. Recuperado de http://www.revistaeducacion.mec.es/re346 11.html

Hansen, W. B. y O’Malley, P. M. (1996). Drug use. EN R. J. DiClemente, W. B. Hansen y L. E. Ponton (Eds.), Handbook of Adolescent Health Risk Behavior. New York: Plenum Press.

Jiménez-Muro, A., Beamonte, A., Marqueta, A., Gargallo, P. y Nerín I. (2009). Consumo de drogas en estudiantes de primer curso. Adicciones, 21, 2128. Recuperado de http://www.adicciones.es/index.php/adicciones/articl e/view/248

La Caixa (2015). El impacto social de las drogas. Hablemos sobre drogas. Barcelona, España: Fundación la Caixa.

Linden, A. N. y Lau-Barraco, C. (2014). A qualitative review of psychosocial risk factors associated with caffeinated alcohol use. Experimental and Clinical Psychopharmacology, 22(2), 144-153. http://dx.doi.org/10.1037/a0036334

Lucena, V., Ruiz-Olivares, R., Pino, M. J. y Herruzo, J. (2013). Consumo de alcohol, tabaco y psicofármacos en jóvenes universitarios y no universitarios. Behavioral Psychology / Psicología Conductual, 21(1), 123-136. 
Martins, S. S., Carlson R. G., Alexandre P. K. y Falck R.S. (2011). Perceived risk associated with ecstasy use: A latent class analysis approach. Addictive Behaviors, 36, 551-554. http://dx.doi.org/10.1016/j.addbeh.2011.01.013

Mas, A., Nerín, I., Barrueco, M., Cordero, J., Guillén, D., Jiménez-Ruiz, C. y Sobradillof, V. (2004). Consumo de tabaco en estudiantes de sexto curso de medicina de España. Archivos de Bronconeumología, 40, 403-408.

Ministerio de Sanidad, Servicios Sociales e Igualdad (2015). Encuesta sobre alcohol y drogas en población general en España. EDADES 2013/2014. Madrid, España: Observatorio Español sobre la Droga y las Toxicomanías, Dirección General para el Plan Nacional sobre Drogas.

Ministerio de Sanidad, Servicios Sociales e Igualdad (2013) Encuesta estatal sobre uso de drogas en enseñanzas secundarias (ESTUDES) 2012/2013, España. Madrid, España: Dirección General para el Plan Nacional sobre Drogas. Recuperado de http://www.msssi.gob.es/gabinete/notasPrensa.do?i $\mathrm{d}=3218$

Morales-Manrique, C., Bueno-Cañigral, F., AleixandreBenavente A. y Valderrama-Zurián J. (2010). Creencias y motivos asociados al consumo y no consumo de cocaína en población escolarizada de la ciudad de Valencia (España). Trastornos Adictivos, $12(2), 58-64$.

Observatorio Europeo de las Drogas y las Toxicomanías, OEDT (2010). El problema de la Drogodependencia en Europa. Informe anual 2010. Luxemburgo, Oficina de Publicaciones de la Unión Europea.

Pino, M. J., Herruzo, C., Raya, A. y Herruzo, J. (2015). Legal and Illegal Substance Consumption and Traffic Accident Risk Perception Among Spanish Young People. Social Indicators Research, 1-11. http://dx.doi.org/11 10.1007/s1120501511214
Ruiz-Olivares, R., Lucena, V., Pino, M. J. y Herruzo, J. (2010). Análisis del consumo de drogas legales como el alcohol, el tabaco y los psicofármacos y la percepción del riesgo en jóvenes universitarios. Psychology, Society, \& Education, 2(1), 21-31.

Ruiz-Olivares, R., Lucena, V., Pino M. J., Raya, A. y Herruzo, J. (2010). El consumo de cannabis y la percepción del riesgo en jóvenes universitarios. Behavioral Psychology/Psicología Conductual, 18(3), 579-590.

Scott-Parker, B., Watson, B., King, M. J. y Hyde, M. K. (2013). A further exploration of sensation seeking propensity, reward sensitivity, depression, anxiety, and the risky behaviour of young novice drivers in a structural equation model. Accident Analysis and Prevention, 50, 465-471. http://dx.doi.org/10.1016/j.aap.2012.05.027

Tirado, R., Aguaded, J. I. y Marín, I., (2009). Factores de protección y de riesgo del consumo de alcohol en alumnos de la Universidad de Huelva. Salud y Drogas, 9(2), 165-184.

Trujillo, A. M., Forns, M. y Pérez Gómez, A. (2007). Uso de sustancias y percepción del riesgo: Estudio comparativo entre jóvenes de Bogotá y Barcelona. Adicciones, 19(2), 179-190.

Uribe, J. I., Verdugo, J. C. y Zacarías, X. (2011). Relación entre percepción de riesgo y consumo de drogas en estudiantes de bachillerato. Psicología y Salud, 21(1), 47-55. http://www.uv.mx/psicysalud/psicysalud-211/index.html 
